\title{
Halbierte Höhlenbärenfersen aus Westfalen und Süddeutschland
}

\author{
Von KARL BrandT, Frankenhain
}

Mit 1 Abbildung

$\mathrm{Z}$ us a mmenf assung. Es werden fünf halbierte Fersenbeine (Calcaneum) von Höhlenbären aus der Balver-Höhle in Südwestfalen beschrieben. Als Vergleich werden gleiche Fundstücke aus drei verschiedenen Höhlen in Süddeutschland erwähnt. Die Frage ist, ob Fraßreste von Höhlenhyänen oder Artefakte vorliegen. Da an den Fundstücken aus der Balver Höhle keine Fraßspuren nachweisbar sind, wie etwa Eindrücke der Eckzähne, nimmt der Verfasser mit Vorbehalt an, daß es Artefakte gewesen sein könnten, zumal er an den Knochenstücken Hiebspuren von schärferen Steinen wahrzunehmen glaubt. Als evtl. Verwendungszweck können Fellglätter angenommen werden oder aber die distalen, knopfähnlichen abgeschlagenen Enden können vielleicht als Spielknöchelchen oder als Jagdtrophäen verwendet worden sein. Im letzteren Falle evtl. durchlocht als Kette getragen wurden. Sicherlich befinden sich in magazinierten Knochenbeständen der Sammlungen gleiche halbierte Fersenbeine.

$\mathrm{Su} \mathrm{m} \mathrm{m}$ a r y. Five halved heel bones (Calcaneum) of the cave bear are described from the Balver-Höhle in southern Westphalia. Similar finds out of three different caves in southern Germany are mentioned for comparison. The question is whether these remains are the liftovers of a hyena's spoils or artifacts. As there are no traces of feeding damage such as the scratch marks of eye teeth, the author suggests, though with reserve, that these remains could be artifacts. Additionally he believes that the marks of blows delivered by sharp stones are to be made out on the pieces of bone.

If a definite use is sought for the bones they could have been employed as skin posilhers; or they might have been used as play pieces or kept as hunting trophies. They could even have been threaded to make a necklace of some sort. Whatever the explanation similar halved heel bones could surely be found in many old museum collections.

In der reichen Universitätssammlung von Erlangen sah ich auch ein halbiertes Fersenbein (Calcaneum) vom Höhlenbären (Ursus spelaeus), das aus der Petershöhle im badischen Hegau stammt. Bei der anschließenden Exkursion in den Fränkischen Jura wurde auch das Heimatmuseum in Pottenstein (Oberfranken) besucht. Hier waren mehrere halbierte Fersenbeine von Höhlenbären ausgestellt. In beiden Fällen wurden diese im Zusammenhang mit Artefakten aus Stein gefunden.

Solche Fersenbeine waren mir nicht unbekannt, da ich im Verlauf von Jahrzehnten aus der Balver Höhle im Hönnetal (Westfalen) fünf gleiche bearbeitete Fersenbeine gesammelt habe und stets Ausschau nach gleichartigen Fundstücken aus anderen Gegenden hielt. Da nun solche Parallelfunde vorliegen, erscheint es angebracht, über die halbierten Fersenbeine kurz zu berichten.

In der Literatur fand ich in den Bayerischen Vorgeschichtsblättern die gleichen Fersenfragmente aus der Höhle Zahnloch bei Steifling (Fränkische Schweiz) beschrieben mit 10 Figuren auf Tafel 1. Es dürfte dies die erste Arbeit über unser Thema sein. Der Verfasser ist der Ansicht, daß es sich um Artefakte handelt, die zum Verarbeiten von Fellen geeignet gewesen seien, worauf starke Abnutzungsspuren hinweisen.

Wie an den gleichen Funden aus Balve, ist an den Fersenbeinen nicht die Spur von Raubtierzähnen zu sehen, nicht einmal jene typischen Eindrücke der Eckzähne, die häufiger an sicher benagten Knochen pleistozäner Säugetiere zu bemerken sind.

Somit haben wir bisher vier Höhlen, die Fragmente von Fersen der Höhlenbären geliefert haben: Petersfels, Pottenstein, das Zahnloch und die Balver Höhle. Den Vorschlag von Max NäBE: „Gleichwohl dürfte es sich empfehlen, die magazinierten Bestände anderer Höhlengrabungen daraufhin durchzusehen“, (S. 5) kann man nur bekräftigen.

In Balve habe ich selbst drei Fersenbein-Fragmente von Jahren an der Böschung vor der Balver Höhle gefunden. Die zwei anderen habe ich aus Sammlungen erworben, wo sie unerkannt zusammen mit anderen Knochenstücken verwahrt wurden. 




Abb. 1. Fünf halbierte Fersenbeine vom Höhlenbären aus der Balver Höhle in Westfalen. $X 1 / 2$.

Bei der Beschreibung der einzelnen Fersenbeine kommt zum Ausdruck, daß ich geneigt bin, dieselben als von Menschenhand bearbeitet anzusehen, obwohl man auch annehmen kann, es könnten Fraßrückstände von Höhlenhyänen oder anderen pleistozänen Raubtieren sein. $\mathrm{Zu}$ meinen Ausführungen in diesem Bericht empfiehlt H. SchwabedisseN folgende Bemerkung:

„Ob es sich dabei allerdings um echte Werkzeugtypen handelt, ist die Frage. Es können bestimmt immer wiederkehrende Formen auch auf Grund fester Techniken bei der Zerlegung des Tieres und seiner Knochen entstehen. Aus dem Mesolithikum kennen wir Parallelen. Diese so entstehenden Formen können durchaus als Gelegenheitsgeräte benutzt werden, ohne daß echte von vornherein gewollte Werkzeuge vorliegen."

Nun zur Beschreibung dieser "neuartigen“ Knochenfunde, die sich genauer nur auf die aus Balve beziehen kann. Verwendet worden sind sowohl linke als auch rechte Fersenbeine ( 3 linke und 2 rechte), die von jüngeren und älteren Tieren stammen. Das Fundstück Figur 1 unserer Abbildung stammt vom ältesten und kräftigsten Tier. Entsprechend der Reihenfolge auf unserer Abbildung folgt die Beschreibung der einzelnen Stücke.

Nr. 1. Das kräftigste Fragment ist etwa zur Hälfte ringsum durch leichte Einkerbungen und danach erfolgten gewaltsamen Abbruch entstanden. Auf unserer Abbildung ist die Einkerbung auf der linken Seite erfolgt, und so liegt die Abbruchkante rechts, was auch an dem zackigen Ausbruch oben zu erkennen ist. Rechts unten an der Abbruchstelle befindet sich eine fast $2 \mathrm{~cm}$ breite und $0,8 \mathrm{~cm}$ auf die Oberseite hinaufreichende dreieckige geformte Schlifffläche, die durch den Gebrauch des Knochens etwa als Glätter entstanden sein kann. Sonst sind keinerlei Gebrauchsspuren irgendwelcher Art zu beobachten.

Nr. 2. Hieran ist die rechte Seite behauen, um die Abbruchstelle vorzuzeichnen. Infolgedessen ist an der linken Seite der gewaltsame Abbruch zu sehen. Irgendwelche Gebrauchsspuren sind nicht zu bemerken.

Nr. 3. Die Abbruchstelle ist fast ringsum vorgezeichnet worden, nur nicht an der Unterfläche, und so erfolgte der Abbruch nach unten, wie an der entsprechenden Aussplitterung an der Unterfläche zu sehen ist. Am proximalen Ende ist die rechte leisten- 
förmig vorragende Leiste absichtlich entfernt worden (s. Pfeil in Abb.). Gebrauchsspuren sind nicht zu entdecken.

Nr. 4. Ist rundherum vorgezeichnet und dann abgebrochen. An diesem Stück sind einzelne Schläge zur Erzeugung des Bruches besonders deutlich zu sehen. Danach dürfte die Bruchfläche nicht vorgestrichelt, sondern etwa mit einem schärferen Feuerstein geschlagen worden sein. Auch an diesem Stück sind keine Gebrauchsspuren zu entdecken. Es ist möglich, daß dieses Stück nachträglich verbissen wurde.

Nr. 5. Hieran sind keinerlei Vorzeichnungen der Abbruchkanten zu erkennen. Vielmehr ist das distale Ende durch zwei kräftige Schläge (die entsprechende Schlagnegative auf der Oberfläche zurückließen) abgetrennt worden. Der Abbruch erfolgte von links nach rechts (Figur 5), wodurch rechts oben ein größeres Knochenstück mit aussprang. Wiederum sind keine Gebrauchsspuren zu entdecken.

Es ist somit möglich, daß die distalen Enden unserer Fersenbeine von Höhlenbären durch Menschenhand absichtlich abgetrennt vorden sind und zwar nicht durch Vorstichelung, sondern durch Schläge mit etwas scharfkantigen Steinen; die einfaste und leichteste Art, solche und ähnliche Abtrennungen an Knochen, Geweih, Elfenbein usw. vorzunehmen. Da nur an Stück 1 Gebrauchsspuren festzustellen sind, kann es auf die Verwertung des rundlichen, knopfartigen distalen Teils der Fersenbeine angekommen sein, oder aber die hier beschriebenen proximalen Enden waren noch nicht in Gebrauch genommen.

Dafür kann der Befund an den gleichartigen Stücken im Heimatmuseum Pottenstein sprechen, wovon mindestens ein Stück am distalen Ende starke Abrundungsspuren aufweist, die nur durch Gebrauch entstanden sein können. Vielleicht wurden sie zum Glätten der Felle verwendet. Deswegen sind die Pottensteiner Stücke auch als Fellglätter bezeichnet. An diesen Verwendungszweck möchte man nicht so ohne weiteres glauben, denn ein vollständig erhaltenes Fersenbein mit dem rundlichen „Knopf“ am distalen Ende war eben durch diesen Kopf vielleicht zweckmäßiger.

Sollten, wie oben schon erwähnt, die distalen knopfartigen Enden der Fersenbeine verwendet worden sein, so kann man sich aus unserer Sicht diesen Verwendungszweck kaum vorstellen, wenn man nicht an Spielknöchelchen denken will (außer als Glätter). Möglicherweise sind die an den Bruchflächen abgerundeten „Knöpfe“ durchlocht aneinandergereiht als Halsketten (Jagdtrophäen) von Bärenjägern getragen worden. Anhaltspunkte über das genauere Alter unserer Fersenbeine haben wir nicht, weil die Balver Stücke im ausgekarrten Höhlenschutt vor der Höhle gefunden wurden, woraus auch die vielen tausend Artefakte aus Kieselschiefer, weniger aus Feuerstein und Grauwacke, stammen. Da der Höhlenbär gegen Ende der Würm-Kaltzeit nicht mehr auftritt, dürften die Fersenbeine aus der ersten Hälfte dieser Kaltzeit stammen und zu dem weitaus am meisten vertretenen Steinartefakten des Mittelpaläolithikums gehören. Nach den Grabungsberichten von J. ANDree fanden sich namentlich in Schicht 2 des Dechenarmes der Balver Höhle: „Zähne, Hand- und Fußknochen von Ursus spelaeus in sehr großen Mengen, z. T. mit Spuren von Abrollung. “ Unsere Fersenbeinfragmente dürften dem Mittelpaläolithikum, der Kulturstufe des Moustérien, die durch Steinartefakte stark in der Balver Höhle vertreten war, angehören.

Die fünf Fersenbeine aus der Balver Höhle habe ich dem Landesmuseum für Vor- und Frühgeschichte in Münster übergeben.

\section{$\mathrm{Schrifttum}$}

Andree, J.: Das Paläolithikum der Höhlen des Hönnetales in Westfalen, Mannus Bibliothek, 42, S. 42, 1928.

NäBE, M.: Zur paläolithischen Knochenkultur. I. Bearbeitete Höhlenbärenknochen aus dem Zahnloch bei Steifling. Bayerische Vorgeschichtsblätter, 14, 1-8, 1937.

Manuskr. eingeg. 18. 1. 1967.

Anschrift des Verf.: Karl Brandt (aus Herne), 3579 Frankenhain, Unter der Kirche 66. 